\title{
On the Einstein-Stern model of rotational heat capacities
}

\author{
Dahl, Jens Peder
}

Published in:

Journal of Chemical Physics

Link to article, DOI:

$10.1063 / 1.477766$

Publication date:

1998

Document Version

Publisher's PDF, also known as Version of record

Link back to DTU Orbit

Citation (APA):

Dahl, J. P. (1998). On the Einstein-Stern model of rotational heat capacities. Journal of Chemical Physics, 109(24), 10688-10691. https://doi.org/10.1063/1.477766

\section{General rights}

Copyright and moral rights for the publications made accessible in the public portal are retained by the authors and/or other copyright owners and it is a condition of accessing publications that users recognise and abide by the legal requirements associated with these rights.

- Users may download and print one copy of any publication from the public portal for the purpose of private study or research.

- You may not further distribute the material or use it for any profit-making activity or commercial gain

- You may freely distribute the URL identifying the publication in the public portal

If you believe that this document breaches copyright please contact us providing details, and we will remove access to the work immediately and investigate your claim. 


\title{
On the Einstein-Stern model of rotational heat capacities
}

\author{
Jens Peder Dahl \\ Chemical Physics, Department of Chemistry, Technical University of Denmark, DTU 207, \\ DK-2800 Lyngby, Denmark
}

(Received 19 June 1998; accepted 18 September 1998)

\begin{abstract}
The Einstein-Stern model for the rotational contribution to the heat capacity of a diatomic gas has recently been resuscitated. In this communication, we show that the apparent success of the model is illusory, because it is based on what has turned out to be an unfortunate comparison with experiment. We also take exception to the possibility of assigning any meaning to the rotational zero-point energy introduced by the model. (C) 1998 American Institute of Physics.
\end{abstract}

[S0021-9606(98)02448-9]

\section{INTRODUCTION}

In a conceptually interesting article from 1913, Einstein and Stern ${ }^{1}$ discussed the possible existence of a zero-point energy for a quantum-mechanical vibrator. Planck had presented arguments in favor of such a zero-point energy two years earlier and had replaced his first formula for the temperature-dependent energy of a harmonic vibrator, ${ }^{2}$

$$
E_{v 1}=\frac{h \nu}{e^{\frac{h \nu}{k T}-1}}
$$

by a second one, ${ }^{3}$

$$
E_{v 2}=\frac{h \nu}{2} \frac{e^{\frac{h \nu}{k T}+1}}{e^{\frac{h \nu}{k T}-1}}=\frac{h \nu}{e^{\frac{h \nu}{k T}-1}}+\frac{h \nu}{2} .
$$

Einstein and Stern pointed out that when expanded to second order in $h \nu / k T$, the first formula gives $E_{v 1}=k T-h \nu / 2$, whereas the second formula gives $E_{v 2}=k T$. The two formulas lead, however, to the same expression for the heat capacity $^{4}$ of a system of $N$ vibrators, namely,

$$
C_{v}=R \frac{h \nu}{k T} \frac{e^{\frac{h \nu}{k T}}}{\left(e^{\frac{h \nu}{k T}}-1\right)^{2}}
$$

with $N$ being the Avogadro constant and $R$ the gas constant. With an additional factor of 3 this is Einstein's expression from 1907 for the heat capacity of a monatomic solid. ${ }^{5}$

As is well known, Einstein's analysis solved the long standing puzzle of the temperature dependence of the heat capacity of solids. According to the equipartition theory of classical statistical mechanics the heat capacity should have the constant value $3 R$. But in fact it drops from the value $3 R$ at high temperatures to the value 0 at $T=0$, and this is what was accounted for by Einstein's expression.

For a diatomic gas, the heat capacity has a value of about $\frac{5}{2} R$ at ambient temperature. According to classical statistical mechanics, this value is made up of a contribution of $\frac{3}{2} R$ from the translational motion, and a contribution of $R$ from the rotational motion. That the rotational contribution to the heat capacity might decrease from $R$ to 0 by cooling was, however, considered a realistic possibility around 1912. So, to test this possibility Eucken measured the heat capacity of molecular hydrogen at several temperatures between 273 and $35 \mathrm{~K}$, and indeed found that the heat capacity diminished from 4.84 to $2.98 \mathrm{cal} \mathrm{mol}^{-1} \mathrm{~K}^{-1}$ in that interval, ${ }^{6}$ i.e., from 2.44 to $1.50 R$. Soon thereafter, the temperature-dependent heat capacities of several other gases were also measured, in particular by Scheel and Heuse. ${ }^{7}$

Eucken tried to represent his data by an expression of the form in Eq. (3), augmented by a $\frac{3}{2} R$ term. But he found that this led to too shallow a curve for the heat capacity as a function of $T$, in comparison with the experimental curve.

This was the starting point for Einstein and Stern's analysis. Their idea was to tie the problem of accounting for the form of the rotational contribution to the heat capacity of a diatomic molecule to the problem of deciding upon the possible existence of a zero-point energy for a vibrator. They treated the molecule as a rigid rotator, with the rotational energy

$$
E_{r}=\frac{1}{2} I(2 \pi \nu)^{2}
$$

where $I$ is the moment of inertia, and $\nu$ is the frequency of rotation which is supposed to depend on the temperature. For each temperature, they compared the energy of the rotator with that of a vibrator with the same frequency $\nu$, and required that the two energies be the same. For each of the above energy expressions, $E_{v 1}$ and $E_{v 2}$, this uniquely determines $\nu$ and hence $E_{r}$ as a function of $T$. Finally, the heat capacity of a gas of rotators was determined as

$$
C_{r}=N \frac{d E_{r}}{d T}
$$

This leads to two expressions for $C_{r}$ as functions of $T$. Einstein and Stern compared the graphs based on these expressions with Eucken's experimental graph of the rotational contribution to the heat capacity of molecular hydrogen. They found that only the graph related to the expression (2) could be brought in good agreement with the experimental 
graph, and hence concluded that the existence of a vibrational zero-point energy had been made probable.

However, the derivation based on the expression (2) also predicted a rotational zero-point energy. Today we know that this was an incorrect prediction. The Einstein-Stern model has, nevertheless, attracted some recent attention, through the presentation by Milonni ${ }^{8}$ and the subsequent discussion by Malta, Marshall, and Santos. ${ }^{9}$ Milonni rejected the model because of our present knowledge, that a molecule has no zero-point rotational energy. However, the latter authors claim that the agreement with the experimental data can be qualitatively explained in the Wigner representation.

In the present communication, we draw attention to the fact that the experimental curve with which Einstein and Stern compared their theoretical curve was the experimental curve for so-called normal hydrogen, which is a nonequilibrium mixture of ortho- and parahydrogen. When a comparison with a proper system is performed, the success of the Einstein-Stern model vanishes, for any diatomic molecule.

In the following, we briefly review the Einstein-Stern model, using a dimensionless representation rather than adopting the somewhat cumbersome units used in Einstein and Stern's article. We then compare the Einstein-Stern expression for $C_{r}$ with the modern expressions derived from statistical mechanics, and demonstrate the poor agreement between the two descriptions. Finally, we comment on the so-called angular-momentum dilemma and the possible existence of a zero-point energy for rotational motion.

\section{THE EINSTEIN-STERN MODEL}

Let us introduce the rotational and vibrational temperatures, $\Theta_{r}$ and $\Theta_{v}$, respectively, by the usual definitions

$$
\Theta_{r}=\frac{h^{2}}{8 \pi^{2} I k}, \quad \Theta_{v}=\frac{h \nu}{k} .
$$

$\Theta_{r}$ is a constant, whereas $\Theta_{v}$ depends on $T$ through the temperature-dependent frequency $\nu$. Expression (4) for the rotational energy may then be written

$$
E_{r}=\frac{h \nu}{4} \frac{\Theta_{v}}{\Theta_{r}} .
$$

The condition $E_{r}=E_{v 1}$ becomes

$$
\frac{1}{4} \frac{\Theta_{v}}{\Theta_{r}}=\frac{1}{e^{\frac{\Theta_{v}}{T}-1}},
$$

from which we get

$$
\frac{T}{\Theta_{r}}=\frac{\Theta_{v}}{\Theta_{r}}\left[\ln \left(\frac{4}{\Theta_{v} / \Theta_{r}}+1\right)\right]^{-1} .
$$

This equation gives the relation between $T$ and $\nu$, or between $\tau$ and $x$, where

$$
\tau=\frac{T}{\Theta_{r}}, \quad x=\frac{\Theta_{v}}{\Theta_{r}} .
$$

The heat capacity may be written

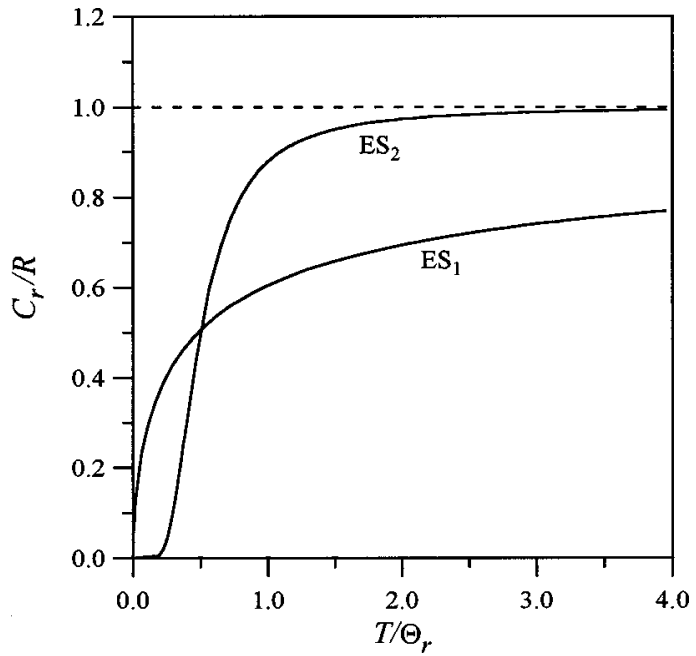

FIG. 1. Molar constant volume heat capacity of a gas of rigid rotators according to the model of Einstein and Stern. " $\mathrm{ES}_{1}$ " is based on expression (12), "ES 2 " on expression (15).

$$
C_{r}=\frac{1}{2} R x \frac{d x}{d \tau},
$$

where $R$ is the gas constant. It leads to the expression

$$
C_{r 1}=R \frac{x^{2}}{2 \tau}\left(1+\frac{\tau}{\frac{x^{2}}{4}+x}\right)^{-1}
$$

which, through Eq. (9), determines $C_{r}$ as a function of $\tau$.

Similarly, the condition $E_{r}=E_{v 2}$ becomes

$$
\frac{1}{4} \frac{\Theta_{v}}{\Theta_{r}}=\frac{1}{e^{\frac{\Theta_{v}}{T}}-1}+\frac{1}{2},
$$

from which we get

$$
\frac{T}{\Theta_{r}}=\frac{\Theta_{v}}{\Theta_{r}}\left[\ln \left(\frac{4}{\frac{\Theta_{v}}{\Theta_{r}}-2}+1\right)\right]^{-1} .
$$

This equation gives the new relation between $\tau$ and $x$ and leads to the alternative expression

$$
C_{r 2}=R \frac{x^{2}}{2 \tau}\left(1+\frac{\tau}{\frac{x^{2}}{4}-1}\right)^{-1}
$$

for the heat capacity.

Figure 1 shows $C_{r 1}$ and $C_{r 2}$ as functions of $T / \Theta_{r}$. As pointed out by Einstein and Stern, the behavior of $C_{r 1}$ for small $T$ is qualitatively incorrect. The graph of $C_{r 2}$ is more convincing, and Einstein and Stern showed that it could be brought in almost quantitative agreement with the graph representing the experimental values of $C_{r}$ determined by Eucken, provided a suitable value was assigned to their parameter $p$. In our notation this parameter is

$$
p=\frac{h^{2}}{4 \Theta_{r} k} \text {. }
$$




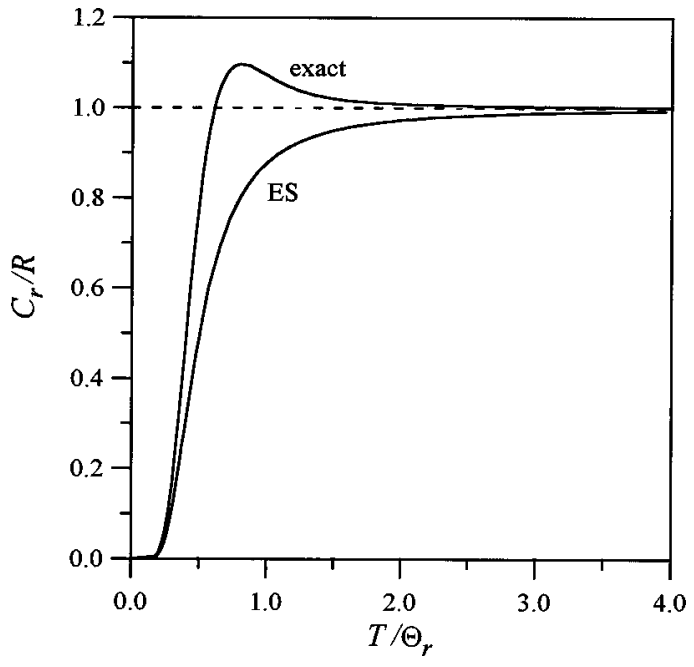

FIG. 2. Molar constant volume heat capacity of a gas of rigid rotators. "Exact" is based on the partition function of Eq. (18), "ES", on the model of Einstein and Stern, expression (15).

For hydrogen, the determined value for $p$ corresponds to $\Theta_{r}=274 \mathrm{~K}$. This is, however, a very unrealistic value, the correct value for hydrogen being $87.5 \mathrm{~K}$, a discrepancy that has so far been left unnoticed.

As already mentioned in Sec. I, the heat capacity curve by Eucken is the curve for so-called normal hydrogen which is a nonequilibrium mixture of ortho- and parahydrogen. In the following section, we shall therefore make a more appropriate comparison.

\section{EXPRESSIONS FOR THE HEAT CAPACITY OF A GAS OF RIGID ROTATORS}

According to modern quantum-statistical mechanics, the heat capacity of a gas of rigid rotators is given by the expression $^{10,11}$

$$
C_{r}=R \frac{\partial}{\partial \tau}\left(\tau^{2} \frac{\partial}{\partial \tau}(\ln q)\right)
$$

where $\tau=T / \Theta_{r}$ as above, and $q$ is the partition function

$$
q=\sum_{j=0}^{\infty}(2 j+1) e^{-j(j+1) / \tau} .
$$

Figure 2 shows the graph of expression (17) together with the graph of expression (15). The two graphs have the same limits for low and for high temperatures, but they approach the limits differently, and the general shapes of the graphs are quite different.

When the rigid-rotator model is used to describe the rotation of molecular hydrogen, the statistics of the nuclear spins must be included. This complication was, of course, unknown to Einstein and Stern whose work was published many years before the discovery of spin. It was first properly discussed by Dennison in $1927 .{ }^{12}$ As is now well known, ${ }^{10,11}$ the presence of the nuclear spins causes the gas to behave like a mixture of two interconvertible gases, orthohydrogen (total nuclear spin one) and parahydrogen (total nuclear spin zero). The partition function for orthohydrogen is obtained by including only odd values of $j$ in the partition function of

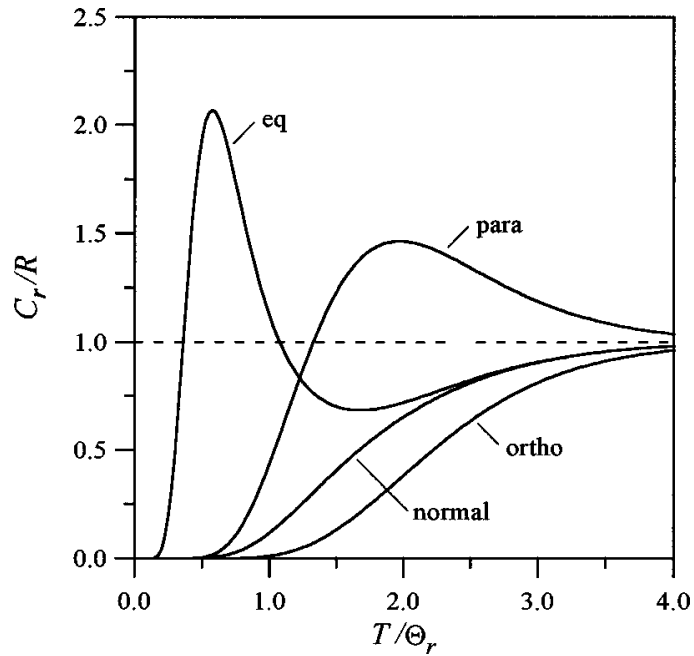

FIG. 3. Molar constant volume heat capacity curves for equilibrated hydrogen, orthohydrogen, parahydrogen, and normal hydrogen.

Eq. (18) and subsequently multiplying by a nuclear-spin degeneracy factor of 3 , while the partition function for parahydrogen is obtained by including only even values of $j$ in the sum of Eq. (18). In catalytically equilibrated hydrogen, the ortho:para ratio varies with temperature, approaching the value 3:1 for large values of $T$. So-called normal hydrogen is a hydrogen gas in which the ratio 3:1 is maintained at all temperatures.

Figure 3 shows the heat capacity curves for orthohydrogen, parahydrogen, normal hydrogen, and equilibrated hydrogen (for which the partition function is $q_{\text {para }}+q_{\text {ortho }}$ ).

In Fig. 4, we show again the heat capacity curve for normal hydrogen, but with the value $87.5 \mathrm{~K}$ inserted for $\Theta_{r}$. Shown are also the Einstein-Stern curves corresponding to $\Theta_{r}=87.5$ and $274 \mathrm{~K}$. Einstein and Stern's analysis corresponds to a comparison of the curve for normal hydrogen at $87.5 \mathrm{~K}$ with the Einstein-Stern $274 \mathrm{~K}$ curve. Although the two curves are quite similar, the result of such a comparison

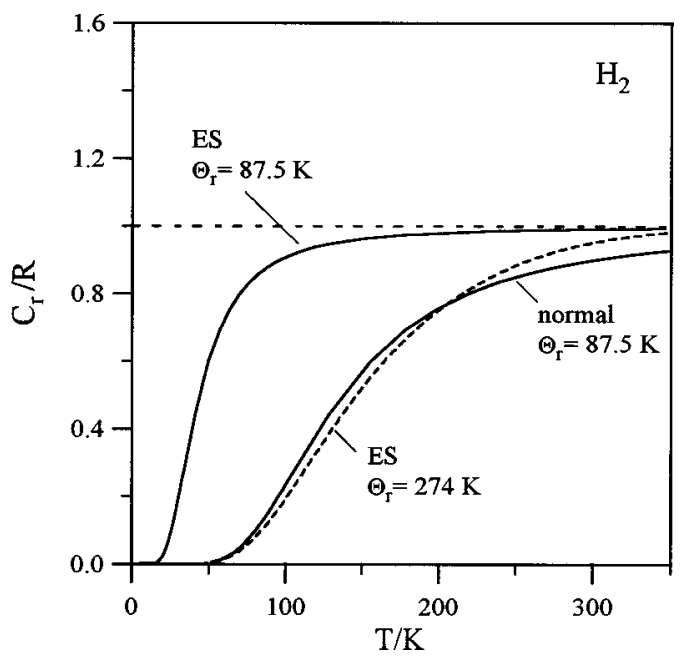

FIG. 4. Molar constant volume heat capacity curve for normal hydrogen compared with heat capacity curves based on the model of Einstein and Stern, expression (15), with $\Theta_{r}=87.5$ and $274 \mathrm{~K}$. 
is now without meaning, and so is accordingly " the remarkable agreement between the curves.'

\section{THE ANGULAR-MOMENTUM DILEMMA}

As mentioned in Sec. I, Einstein and Stern used their analysis to favor expression (2) for the energy of a vibrator over expression (1). In the light of the present work, according to which the Einstein-Stern model in fact leads to an unacceptable heat capacity curve, this becomes a weak deduction. But then it is not needed anyway, since the correctness of expression (2) follows straightforwardly from modern molecular quantum mechanics. Molecular quantum mechanics also uniquely predicts the energy levels of a rigid rotator to be

$$
E_{j}=\frac{h^{2}}{8 \pi^{2} I} j(j+1), \quad j=0,1,2, \ldots
$$

as already used in setting up the partition function of Eq. (18). Expression (19) leads to a vanishing zero-point energy for the rotator.

Relation (13) leads to $\Theta_{v}=2 \Theta_{r}$ at $T=0$. It then follows from the relations (6) and (7) that the Einstein-Stern model predicts a rotational zero-point energy

$$
E_{r 0}=\frac{h^{2}}{8 \pi^{2} I} .
$$

In light of our rejection of the validity of the Einstein-Stern model, we need not assign any specific meaning to this result. Yet, we must comment on the remark made by Malta et al., ${ }^{9}$ that it can be qualitatively explained in the Wigner representation.

We have discussed the Weyl-Wigner representation and applications of it in several articles. In connection with our work on the electronic Wigner function for the ground state of the hydrogen atom, ${ }^{13}$ we discussed what we called the angular-momentum dilemma at some length. This is the problem of reconciling the fact that the Bohr theory predicts an angular momentum different from zero for the hydrogen atom's ground state, with the fact that the Schrödinger theory predicts the value zero. As we pointed out in our article, the difference can be qualitatively understood by realizing that the Weyl transform of the operator $\hat{\ell}^{2}$ is the phase-space function $\ell^{2}-\frac{3}{2} \hbar^{2}$, as first noticed by Shewell. ${ }^{14}$ Thus, the zero expectation value of the operator $\hat{\ell}^{2}$ necessarily corresponds to a nonvanishing phase-space average $\frac{3}{2} \hbar^{2}$ of the function $\ell^{2}$. In their article on the Wigner density of a rigid rotator, Malta et al. turn this argument upside-down by assigning an a priori interest to the phase-space function $\ell^{2}$. This, then, let them suggest that the proper angular momentum operator be $\ell^{2}+\frac{3}{2} \hbar^{2}$ rather than simply $\ell^{2}$. As a result, the rotator acquires an angular momentum of $\frac{3}{2} \hbar^{2}$ in its ground state, which in a qualitative way is supposed to explain a nonvanishing zero-point energy. There is, however, no doubt that Eq. (19) is the correct expression for the energy of a molecular rotator and hence, that the operator $\hat{\ell}^{2}$ correctly accounts for the magnitude of the angular momentum of a rotator.

\section{CONCLUSIONS}

In the present work, we have shown that the success of the Einstein-Stern model is an illusion, and we have eliminated the incentive to seek a justification of a nonvanishing zero-point energy for rotational motion.

\section{ACKNOWLEDGMENT}

This work was supported by the Danish Natural Science Research Council.

\footnotetext{
${ }^{1}$ A. Einstein and O. Stern, Ann. Phys. 40, 551 (1913).

${ }^{2}$ M. Planck, Ann. Phys. 4, 553 (1901).

${ }^{3}$ M. Planck, Ann. Phys. 37, 642 (1912).

${ }^{4}$ Throughout the paper, heat capacity is understood to mean molar constant volume heat capacity.

${ }^{5}$ A. Einstein, Ann. Phys. 22, 180 (1907).

${ }^{6}$ A. Eucken, Sitzungsber. der Königl. Preuss. Akad. der Wissensch., 1912 (unpublished), p. 141.

${ }^{7}$ K. Scheel and W. Heuse, Ann. Phys. 40, 473 (1913).

${ }^{8}$ P. W. Milonni, The Quantum Vacuum (Academic, New York, 1994), Sec. 1.10 .

${ }^{9}$ C. P. Malta, T. S. Marshall, and E. Santos, Phys. Rev. E 55, 2551 (1997).

${ }^{10}$ G. S. Rushbrooke, Introduction to Statistical Mechanics (Oxford University Press, London, 1949), Chap. 7.

${ }^{11}$ R. S. Berry, S. A. Rice and J. Ross, Physical Chemistry (Wiley, New York, 1980), Sec. 21.5.

${ }^{12}$ D. M. Dennison, Proc. R. Soc. London, Ser. A 45, 483 (1927).

${ }^{13}$ J. P. Dahl and M. Springborg, Mol. Phys. 47, 1001 (1982).

${ }^{14}$ J. R. Shewell, Am. J. Phys. 27, 16 (1959).
} 